\title{
Hommages à Olivier Grignon
}

\section{WK L'analyse, passion intraitable}

Olivier Grignon fut, autant qu'il m'en souvienne, l'ami le plus fidèle que j'eusse de toute mon existence.

Contrairement à bien des amitiés trop amoureuses qui se retournent vite en haine ou en rivalité, jamais il n’y eut entre nous ce genre de retournement. Dès l'instant où nous nous réunîmes autour du référé (en 1980) pour combattre la dissolution de l'EFP et que nous nous unîmes aux trois autres fondateurs pour créer le Cercle freudien en 1982, aucune ride ni orage n'eut jamais lieu entre nous, en dépit de toutes les dissensions que la vie d'une association peut susciter. Olivier n'était pas homme à se laisser emporter par la jalousie, la rivalité ni l'envie. La générosité la plus simple, la plus droite et la plus naturelle l'habitait. Cette générosité était je crois sa qualité dominante, qui engendrait toutes les autres, y compris son talent d'analyste. Dans sa façon d'être analyste, en effet, il y avait une grande douceur, une grande intelligence et un grand respect, et cette façon se traduisait dans sa façon d'être au monde, avec ses amis. Nous nous retrouvions dans cet attachement à l'expérience analytique, à l'encontre de tout psitacisme, et si nous ne récusions pas l'enseignement lacanien dont nous étions issus, c'était au profit d'une libre recherche, hors de toute allégeance de langage et de pensée susceptible de conduire à une dogmatique rigide ou de reposer sur un canon préformé. Les échanges autour de cette libre recherche où nous nous confortions l'un l'autre furent assurément ce qu'il y eut de plus précieux et de plus stable dans notre amitié. Une confiance quasi absolue l'un envers l'autre nous unissait dans cette amitié bien tempérée née de la pulsion analytique...

Une blessure secrète, néanmoins, une fêlure, était en lui, un fond mélancolique qui soutenait à l'arrière-plan cette générosité et lui donnait sa résonance musicale et sa profondeur charnelle : par son titre même, Le corps des larmes (paru en 2002) témoignait de l'inoubli d'une douleur où s'enracinait sa joie de vivre. Car Olivier était aussi un bon vivant et un gai compagnon. Il aimait les grands espaces et les voyages en pays magiques, Bali, l'Inde, l'Egypte - que nous parcourûmes ensemble dans une sorte de baptême du Cercle, à la fin la Martinique, où il semait encore la psychanalyse lorsque la maladie l'attrapa ; les crêtes et les montagnes, les alpages où il fit l'une de ses demeures, au point d'y apprendre l'apiculture pour la mieux mériter. C'était un 
Tibet reconstitué. Sa modestie tenait à cette confrontation permanente avec plus grand que lui.

Le corps des larmes offrit alors une théorie intense de la psychanalyse, dont le modèle était à chercher dans la glisse, selon la définition donnée par Deleuze : “ insertion sur une onde préexistante ». Dans la glisse en effet, "il n’y a fixation ni sur le départ ni sur l'arrivée, mais seulement du mouvement, des rapports ", selon une autre formule empruntée à J.-L. Godard. Et c'est ainsi qu'Olivier pensait la cure analytique, sur le mode d'une onde préexistante sur laquelle nous étions greffés, les uns et les autres (Lacan et Freud compris), de manière à constituer une communauté d'expérience. Ce qui n'allait pas sans la nécessité d'incarner « une érotique historiquement repérable " : "Car ce qui distingue une époque et définit une contemporanéité, c'est une communauté d'expérience, voire une érotique historiquement repérable », écrivait-il. C'est l'esprit d'une telle communauté que nous tentions d'insuffler dans la vie du Cercle freudien, en dépit des résistances qui nous valurent certaines inimitiés, et dont il reprit le flambeau au cours de sa récente présidence du Cercle.

Si les arts de la glisse sont des arts de l'extrême et des passions intraitables, la psychanalyse pour lui l'était aussi, réclamant un travail à proportion : “ Inscrire de telles expériences dans le corpus de la psychanalyse réclame un labeur obstiné avant de pouvoir les qualifier de privilégiées ", écrivait-il. Ainsi, Olivier Grignon plaçait-il résolument la psychanalyse du côté de l'art et des artistes, et de leurs expériences obstinées, comme de leurs audaces solitaires. Une passion, donc, traversait et relançait à chaque instant l'intelligence de cette pulsion extrême qui l'animait, onde branchée sur une onde, passion intraitable, qui se transmettait pour tenter d'atteindre à la sublimation commune.

La corrida était, avec la glisse, l'autre référence majeure du livre : la force animale, avec son intelligence obscure, parfois stupéfiante ${ }^{[7]}$, s'avérait le contrepoint nécessaire de notre humanité, de son émergence radicale face à la mort. Avec l'oubli de la mort, nous sommes aussi dans l'oubli de l'animal. L'art extrême de la psychanalyse ne va donc pas sans un risque vital : ce risque cherche son équilibre précaire, puisqu'il n'y a fixation ni sur le départ ni sur l'arrivée, la psychanalyse ne se définissant pas plus par son achèvement que par le symptôme qui en serait l'origine, autour d'un terme mobile, celui de “Passe », que nous avions hérité d'une expérience ratée de l'EFP, au dire de Lacan lui-même, quoique cruciale (puisqu'on y pouvait lire, aussi bien, une des raisons de la fin de l'EFP). Terme où l'on entendra, à côté de la proposition de Lacan, tant la passe dangereuse du torero que la passe risquée du surfeur. Signifiant oscillant et précaire plutôt que terme fixe ou levier, comme si le travail d'insertion sur l'onde glissante de l'expérience devait trouver un jour ou l'autre le rituel éphémère et dangereux de sa consécration. C'est à la recherche de cette passe, en somme, 
qu'Olivier Grignon aura consacré les dernières années de sa vie, dont il souhaitait vivement laisser le témoignage écrit et rassemblé, si la mort ne l'avait pris de court. Mais nous, restons avec une question. Celle de la psychose et de son clivage. Si la psychose est l'onde de base, l'onde porteuse qu'il s'agit d'universaliser à partir de son expérience-limite, pour la sortir de la pathologie, de quelle psychose s'agit-il ? Dans le sens, sans doute, de son expérience la plus intime, Olivier Grignon semble avoir donné, à la suite de Lacan, le pas à la mélancolie, privilégiant la “ douleur d'exister ». "On comprend que pour un psychanalyste, avec la psychanalyse, ça devient plus ou moins rapidement un mariage de raison, et que même, parfois, il y a une phase mélancolique de lien à la psychanalyse ", affirmait-il dans Perspectives 2 (conférence du 3 octobre 2011). La " Passe », dénuée cependant de toute procédure institutionnelle, demeurait pour lui une aventure singulière, exceptionnelle quoiqu'exigible de l'analyste : “ un état de sujet extrêmement archaïque » (Perspectives 1, conférence du 19 décembre 2007). “ Je remarque seulement que Lacan pensait qu'il faut cet état de sujet-là pour entendre, pour entendre quelque chose d'incroyablement archaïque. » (ibid., 2007) Tel était donc l'axe, pour lui, la visée de la “ Passe » telle qu’il l'entendait: ce " quelque chose d'incroyablement archaïque », cet " état de sujet » extrême qui aurait pu faire néanmoins " communauté d'expérience ».

“Or nous sommes aujourd'hui confrontés à une catastrophe ; cette catastrophe, c'est que Lacan ne s'exporte pas ou ne se diffuse pas ... ou pire, il se diffuse au pire. " (ibid., 2011) “ Aujourd'hui où Lacan est introduit en Chine ou ailleurs par des énormes machines psychanalytiques, c'est justement ce Lacan trahi, où on confond la fin avec les moyens. » (ibid., 2011) “ Il y a donc des excès néfastes et qui sont hélas, comme je le disais, la façon dont l'œuvre de Lacan se répand. » (ibid., 2011)

Il semblerait, dans cette perspective, qu'Olivier ait suivi une pente de plus en plus pessimiste, non point désabusée, mais de plus en plus désespérée, à en croire par exemple cette remarque : "Je voulais en venir à ça en raison du constat évident, lancinant, mais qu'il faut décomposer entre toutes ces composantes, que la psychanalyse nous pèse ; elle nous pèse terriblement. Simplement, elle ne nous pèse pas de la même façon selon le point où on en est de notre rapport avec la psychanalyse, même si la plupart du temps, ça nous pèse. " (ibid., 2011) N'était-ce pas que la “ communauté d'expérience » tant désirée lui apparaissait dès lors comme de plus en plus improbable, hors de portée même de cette pointe extrême de « l'incroyablement archaïque »?

Etait-ce sa pente mélancolique qui l'entraînait dans la suite d'un certain sillage lacanien ? Le sentiment d'une solitude croissante? La ruine progressive et la dissémination définitive à ses yeux de cette communauté improbable? S’il est vrai que la psychose, la confrontation à sa clinique, furent bien le substrat de la pensée analytique dans toute la seconde moitié du $\mathrm{XX}^{\mathrm{e}}$ siècle, il n’en reste pas moins que les options fondatrices divergeaient, de Lacan à Deleuze, par exemple, qui, lui, procédait 
à partir de la schizophrénie. Jean Oury lui-même se référait tout autant à la phénoménologie et à la psychothérapie institutonnelle qu'à la psychanalyse lacanienne. Les conséquences en sont considérables, notamment quant aux rapports du sujet au collectif. A partir de l'expérience mélancolique en effet, il est particulièrement difficile d'instituer un collectif, voire même de le penser, comme en témoignent les tentatives de Maurice Blanchot ou de Jean-Luc Nancy pour surmonter cette difficulté. En revanche, à partir de l'expérience schizophrénique, le sujet se trouve plongé d'emblée dans le divers, en proie au bruit de fond du monde, assigné, comme disait Deleuze, à halluciner les mots et les choses, et non point à ruminer la perte d'un objet inaccessible, jusqu'à y trouver son persécuteur. Ce fut, peut-être, d'ailleurs, un point crucial dans la divergence lacanienne avec Derrida, qui avançait sur les pas de la dissémination.

Alors que pour Deleuze et Guattari, la paranoïa représentait la mauvaise face de la psychose, celle qui se place et agit du côté du pouvoir et de la répression, Lacan, depuis son emprunt de la " connaissance paranoïaque " à Salvador Dali, a donné à la paranoïa un privilège complétant celui de la mélancolie, sous une double forme, comme le rappelle Olivier Grignon dans son livre : d'une part l'affirmation d'une " paranoïa primordiale », d'autre part la conception de la cure comme " paranoïa dirigée » (qui s'opposait donc terme à terme à la schizo-analyse).

Ce "Lacan trahi », qu'était-il donc, à la fin, pour Olivier? L'horizon d'une exigence thérapeutique de plus en plus affirmée pour la psychanalyse. "Je tiens à affirmer les choses de la façon la plus simple qui soit, même si c'est une façon tout à fait incorrecte, que c'est la psychanalyse qui guérit, parce que c'est elle qui va au plus profond. [... Aujourd'hui, cette psychologisation massive de la psychanalyse s'autorise d'un démenti : le démenti du soin, die Verleugnung du soin. "(Perspectives 1, 2007). C'était un combat solitaire et sans fin assignable, avec peu de secours, contre les tendances les plus marquées d'un " refus de guérir » dans un univers analytique dès lors replié sur soi et sa rumination. Il y avait assurément, dans cette volonté thérapeutique d'Olivier Grignon, une volonté de maintenir l'ouverture de la psychanalyse sur le monde réel, et pour tout dire l'affirmation d'un « savoir désirable » dont il est de la responsabilité des psychanalystes eux-mêmes de maintenir l'existence.

Or, au vu des dérives actuelles d'un certain lacanisme rigide, contre lesquelles Olivier portait déjà des mots furieux, mais encore incantatoires - ainsi : “ qu'elle [la psychanalyse] travaille sans relâche à ne pas devenir l'outil réactionnaire privilégié des gardiens du trône et de l'autel ! »- on peut se demander si ces dérives ne tiennent pas précisément aux décisions fondamentales que je viens d'indiquer concernant la psychose. Si la “ mélancolisation » de la psychanalyse lacanienne est, de fait, inséparable des privilèges accordés à la paranoïa, on comprendra que certains analystes lacaniens se soient eux-mêmes promus gardiens d'un ordre symbolique, à titre de rempart contre la menace d'effondrement mélancolique. Et si l'on pense que se fomente 
une guerre entre mélancolie et schizophrénie, entre abîme subjectif de la “ douleur d'exister » et transformations des normes sociales selon l'esprit du temps, n'est-il pas logique de croire que pour contrer certaines évolutions malignes, il convient de dresser le mur d'un " ordre symbolique », qui toujours fera préférer l'Un antérieur au multiple à venir? Olivier lui-même restait neutre face aux débats où je m'étais engagé d'autre part.

Tels sont quelques échos, qui ne cesseront de résonner en moi, de la passion intraitable d'Olivier Grignon pour la psychanalyse.

Claude Rabant

31 janvier 2014

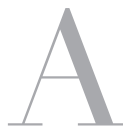

u retour des vacances, début septembre 2013, Olivier Grignon nous a quitté.

Lucide, jusqu'au bout, sur l'issue fatale qui l'attendait, il n'a cédé en rien sur sa passion de transmettre la psychanalyse ni sur son goût pour la bonne chair, le vin et l'amitié. Il a su et voulu, également, résister à une forme d'écrasement provoqué par la médecine dans son acharnement à pousser le soin toujours plus loin, parfois au-delà du supportable. Lutter contre toutes formes d'écrasement intérieur ou extérieur qui guettent tout un chacun a, sans doute, été un des axes dans son travail. Un autre axe a été le souci de maintenir un certain pragmatisme dans son travail clinique mais également dans son rapport à la théorie. Son séminaire mené durant plusieurs années sur le rêve était l'occasion pour lui d'interroger ce sur quoi, Freud et surtout Lacan s'appuyaient dans leur expérience d'homme pour être psychanalystes. Olivier a travaillé au Cmpp de Fontainebleau pendant 28 ans de 1982 à 2010. Il en a été le directeur durant 17 ans. Ce fut une grande chance de travailler avec lui, et ceci pour plusieurs raisons. Tout d'abord parce qu'il a toujours su oeuvrer pour que ce lieu soit le plus convivial possible. Sa convivialité naturelle a diffusé à l'ensemble de l'institution.

Une autre raison qui a contribué à cette chance est d'avoir pu le côtoyer en tant que clinicien. Olivier avait une intelligence clinique, pleine de tact et de respect de l'autre, qu'il aimait partager. Il avait d'ailleurs en lui un formidable désir de transmettre.

Cette grande pertinence clinique tenait, bien entendu, à son expérience de psychanalyste et à son savoir théorique mais elle tenait également à ses qualités d'homme, à l'homme qu'il était.

C'était quelqu'un de très sensible à tout ce qui pourrait mettre à mal la fragilité ou la vulnérabilité de l'humain. Il était très important, pour lui, de respecter cette fragilité cette vulnérabilité car elles représentaient à ses yeux promesses de capacité créatrice pour chacun. C'est pourquoi, il fallait, selon lui, résister, résister à une certaine 
dérive oppressante de nos sociétés modernes, dérive oppressante et conformiste qui s'abat depuis un moment sur les lieux de soin tels que les Cmpp, mais aussi sur bien d'autres lieux de soin prenant en charge la souffrance psychique.

Dans son idée, semble-t-il, le Cmpp de Fontainebleau était une enclave de résistance, une sorte de village gaulois face à un monde où il faudrait marcher tous du même pas. Par son action, en tant que médecin directeur, il permit aux différents thérapeutes de se tenir à l'abri de cette dérive et de pouvoir ainsi exercer leur art avec la tranquillité et la liberté nécessaire.

Patrick Belamich 